\title{
Modelo de Alineación de Prótesis Transtibiales usando Teoría de la Información
}

\author{
Model of Transtibial Prosthesis Alignment with Information Theory
}

\author{
L. A. Luengas-Contreras iD ; P. R. Vizcaya- Guarín iD ; G. Sánchez- Prieto iD
}

\begin{abstract}
Amputation is the removal of a limbs or other appendage or outgrowth of the body. Transtibial amputation or Below-knee amputation (BKA) is a surgical procedure performed to remove the lower limb below the knee when that limb has been severely damaged or is diseased. BKAs are performed due to peripheral vascular disease, disease of the circulation in the lower limb, trauma or congenital. The goal of rehabilitation is return to normal activity. This goal is often accomplished with the help of a prosthesis coupled with gait training. Static alignment of the prosthesis affects the amputee's comfort and the use it of this method of physical rehabilitation, but this process tends to be subjective, lacking information on affectation of alignment over the biomechanical parameters of the subjects. Stochastic predictive model based on knowledge about the prostheses' static alignment was created. First, individuals with unilateral transtibial prostheses were evaluated to investigate the effect of prosthetic alignment changes on lower limb angles, weight bearing and location of center of pressure (COP) when the subject is in the static standing up position. Second, the data analysis of measurements was made and third, the model construction was made taking the information theory as a basis. KolmogorovSmirnov (KS) test and Kullback-Leibler (KL) divergency were used to validate models, accuracy of more than $84 \%$ and a length of the maximum distance of 0.36 bits was obtained, evidencing that data are correctly adjusted to the model obtained.
\end{abstract}

Index Terms-Information theory, Static Alignment, Stochastic model, Transtibial prostheses.

Resumen-La amputación transtibial es la extirpación del segmento corporal por debajo de rodilla, se presenta por accidentes, malformaciones congénitas o enfermedades, tiene repercusiones físicas, biológicas y psicológicas en el individuo amputado. Una forma de ayuda para el amputado es la rehabilitación física, allí se adapta una prótesis mediante la alineación para que supla morfológica y funcionalmente el segmento perdido. La alineación estática de prótesis influye en la comodidad del amputado e incide directamente en el uso o no de este método de rehabilitación física pero este proceso tiende a ser subjetivo, falta información de la afectación de la alineación sobre parámetros biomecánicos de los sujetos. Esta investigación propone un modelo predictivo estocástico, basado en conocimiento, de la alineación estática de prótesis. Inicialmente fueron medidos sujetos amputados transtibiales usuarios de

Este manuscrito fue enviado el 31 de marzo de 2018 y aceptado el 26 de mayo de 2019.

L. A. Luengas-Contreras, docente investigadora Universidad Distrital Francisco José de Caldas, Bogotá, Colombia (laluengasc@udistrital.edu.co). prótesis para establecer los efectos de la alineación de la prótesis transtibial sobre los ángulos de las extremidades inferiores, distribución de peso sobre los pies y ubicación del Centro de Presión (COP) cuando el sujeto se encuentra en posición de bipedestación estática. Luego se analizaron los datos de las mediciones y posteriormente se construyó el modelo tomando como base la teoría de la información. El desempeño del modelo se midió haciendo uso de la prueba de Kolmogorov-Smirnov (KS) y de la divergencia de Kullback-Leibler (KL), se obtuvo un rendimiento superior al $84 \%$ y una longitud de la distancia máxima de 0.36 bits, lo cual evidencia que los datos se ajustan correctamente al modelo desarrollado.

Palabras claves - Alineación estática, Modelos estocásticos, Prótesis transtibiales, Teoría de la Información.

\section{INTRODUCCIÓN}

$\mathrm{L}$ AS personas amputadas alrededor del mundo son más de 10 millones [1], las minas antipersona han afectado a más de 300 mil personas en el mundo y han generado cerca de 26 mil amputaciones [2]. Colombia se encuentra en el proceso de desminado humanitario luego de más de nueve mil heridos a causa de este tipo de artefacto explosivo [3], varios de ellos con amputación transtibial, es decir con pérdida del segmento corporal por debajo de rodilla.

Una prótesis es una extensión artificial que reemplaza o provee una parte del cuerpo faltante, debe realizar la misma función que un segmento natural y lograr un equilibrio estético. La prótesis se utiliza como medio de rehabilitación de los amputados, para su adecuado uso se requiere la adaptación entre este elemento y el sujeto logrando la alineación [4]. En la práctica clínica, la alineación de prótesis es un proceso observacional iterativo que consume tiempo, es subjetivo y requiere muchos años de experiencia del personal calificado que la realiza [5], [6]. Los cambios de la alineación a la prótesis transtibial en el plano sagital influyen en la comodidad del amputado en posición de bipedestación erguida [7]. La información acerca de las variables biomecánicas de rangos articulares, distribución de fuerza sobre los pies y ubicación del COP como consecuencia de la función biomecánica de la prótesis son una manera de hacer objetiva la alineación estática

P. R. Vizcaya- Guarín, docente investigador Pontificia Universidad Javeriana, Bogotá, Colombia (pvizcaya@ javeriana.edu.co).

Giovanni Sánchez-Prieto, Director Tecnologías Universidad de San Buenaventura, Bogotá, Colombia (giosanpri@gmail.com). 
de prótesis.

La influencia la alineación sobre parámetros biomecánicos ha sido probada. Al alterar la alineación en el plano sagital, la compensación del amputado transtibial es el balanceo de la parte superior del cuerpo sobre el centro de presión del pie protésico [8]. La alineación angular tiene efecto significativo en la distribución de la presión plantar y en la magnitud de la presión en el lado protésico [9]. La posición del encaje influye en los momentos de reacción presentes en el muñón en el plano sagital y el coronal [7]. La distribución del peso es significativamente diferente en amputados y no amputados, los ángulos de las rodillas y del tronco son mayores en amputados [10].

No se tienen estudios de modelos que permitan observar la influencia de la posición de los componentes de la prótesis sobre parámetros cinemáticos o cinéticos presentes en la alineación estática en pacientes amputados transtibiales.

Teniendo en cuenta la necesidad de contar con herramientas tecnológicas que apoyen el proceso de alineación de prótesis, se realizó una investigación con el fin de caracterizar parámetros biomecánicos en la alineación estática de prótesis transtibiales. Para ello, en primera instancia se creó una base de datos de la afectación de la posición angular del encaje en el plano sagital sobre variables biomecánicas del amputado; en segunda instancia se desarrolló un modelo estocástico, basado en el conocimiento, que muestra las reacciones de las variables biomecánicas cuando el ángulo del encaje varía.

La estructura planteada para este documento es: Metodología muestra cada una de las etapas desarrolladas en la investigación; Resultados y Discusión explica cómo se planteó y desarrolló el modelo biomecánico de la alineación estática y se incluye el análisis de datos; Conclusiones presenta los hallazgos y la contribución del proyecto.

\section{Metodología}

La obtención de la base de datos se realizó a través de un estudio descriptivo transversal con componente observacional donde se hizo la medición de variables biomecánicas de rango articular de segmentos inferiores, ubicación del COP y distribución de peso bajo cada pie, con siete diferentes valores de ubicación sagital del encaje de la prótesis, a sujetos amputados transtibiales que son usuarios de prótesis.

El estudio fue avalado por el Comité de Ética del Hospital Militar Central, Bogotá, Colombia.

\section{A. Sujetos}

Las personas involucradas en el estudio fueron pacientes amputados transtibiales debido a trauma por minas antipersonas, todos hombres con amputación transtibial unilateral con edades que oscilan entre 29 y 40 años (media de $33.29, \pm 3.77$ años), masa corporal entre 67 y $76 \mathrm{~kg}$ (media de $71 \pm 4.15 \mathrm{~kg}$ ), estatura entre 160 y $176 \mathrm{~cm}$ (media de $171, \pm 0.5$ $\mathrm{cm}$ ), con uso adecuado de prótesis por más de un año, con adaptación adecuada. El tipo de prótesis utilizada es con sistema de suspensión por encaje interno y perno (liner y pin en inglés), y pie de alta actividad en fibra de carbono. El criterio de exclusión de los sujetos fue la presencia de alteraciones músculo esqueléticas o neurológicas en las otras extremidades, alteraciones sensoriales o cognitivas, lesiones en piel, alteraciones en marcha secundarias por dolor, uso de ayudas externas para la marcha, alteraciones articulares en otros segmentos corporales en miembros inferiors.

\section{B. Instrumentos}

La medición de rangos articulares se realizó con el Sistema de Laboratorio de Adquisición de Datos, tipo No. LS900 de la empresa Biometrics Ltd®, que consta de goniómetros digitales de doble eje, un sistema de adquisición de datos (referencia DLK900), software de adquisición y tratamiento de señales DataLINK DLK900 versión software 5.0 [11]. La medición de fuerza plantar se realizó con el sistema Pedar ${ }^{\circledR}$, consiste en plantillas instrumentadas que permiten obtener información de la fuerza que interactúa entre el pie y la suela del zapato, así como la medición de la distribución de la fuerza de contacto y la ubicación del centro de presión (COP); este sistema tiene 198 sensores capacitivos (99 en cada plantilla), software y sistema de visualización, es producido por la empresa Novel [12].

\section{Protocolo}

Las variables utilizadas en el estudio fueron de identificación (Número de identificación), Antropométricas (Edad, Talla, Peso, Tiempo de amputación), Variable de entrada (ANGULO, posición angular del encaje en plano sagital), Variables de salida (variables biomecánicas presentes en la alineación estática: ángulo de las articulaciones ipsilaterales y contralaterales, porcentaje de peso soportado en cada pie, ubicación del COP en cada pie).

El estudio fue desarrollado utilizando guías 2D para controlar la posición de los pies y de bipedestación durante la toma de muestras. La Fig. 1 muestra el uso de las guías (recuadro en frente de los pies del sujeto) y la ubicación de los equipos de medición. Las guías colocan los pies del sujeto con puntos medios del talón a $150 \mathrm{~mm}$ de distancia y con un ángulo de progresión de $8^{\circ}$ [13].

La alineación inicial de la prótesis se consideró óptima y el ángulo del encaje de $0^{\circ}$. Se realizaron seis cambios en el ángulo sagital del encaje $\left(2^{\circ}, 4^{\circ}\right.$ y $6^{\circ}$ en flexión, $2^{\circ}, 4^{\circ}$ y $6^{\circ}$ en extensión) a partir del valor óptimo de alineación. El cambio angular se midió con un goniómetro digital. Se midieron siete condiciones de ubicación angular sagital del encaje, Figura 1. Cada sujeto se instruyó para que permaneciera en posición de bipedestación erguida estática durante 10 segundos. Se realizó la medición de las variables de salida con cada ubicación del encaje. El experimento se repitió tres veces con cada sujeto, el intervalo de tiempo entre mediciones fue superior a un día, de esa forma se recolectaron datos o conjuntos de datos de tres sesiones. 


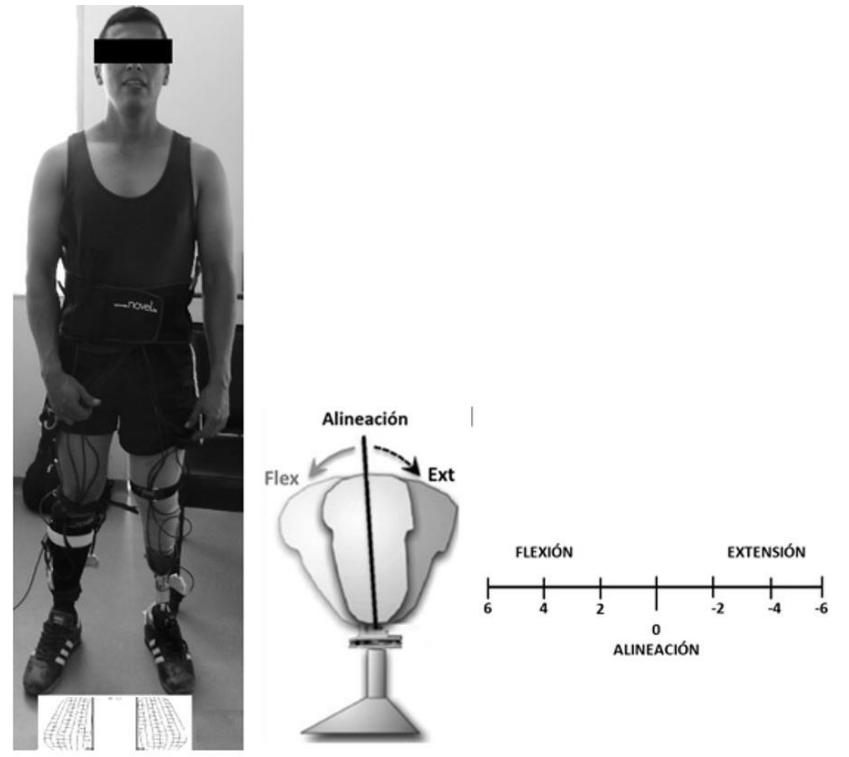

Fig. 1. Sujeto con los equipos para realizar las mediciones de rangos articulares, distribución de peso en cada pie y ubicación del COP en las plantas de los pies en cada una de las siete ubicaciones del encaje. El cuadro blanco enfrente de los pies es la guía 2D usada para controlar posición de los pies.

\section{Análisis de Datos}

Los datos fueron extraídos en archivo plano, luego almacenados en EXCEL $\AA$ donde se codificaron las variables. Posteriormente se trataron para eliminar datos atípicos. Se obtuvieron los valores estadísticos de tendencia central y dispersión de las variables involucradas en el estudio, Tabla I.

TABLA I

VALORES DE TENDENCIA CENTRAL Y DISPERSIÓN

\begin{tabular}{lccccc}
\hline \hline \multicolumn{1}{c}{ VAR } & Rango & Mín & Máx & $\overline{\boldsymbol{X}}$ & $\mathrm{S}$ \\
\hline ANGULO $\left(^{\circ}\right)$ & 12 & -6 & 6 & 0 & 4 \\
$\mathrm{CNA}\left({ }^{\circ}\right)$ & 4,7 & -2 & 2,7 & $-0,11$ & 1,49 \\
$\mathrm{RNA}\left({ }^{\circ}\right)$ & 5,1 & -1 & 4,1 & 2 & 1,43 \\
$\mathrm{TNA}\left({ }^{\circ}\right)$ & 7,4 & $-0,9$ & 6,5 & 3,16 & 2,02 \\
$\mathrm{CDA}\left({ }^{\circ}\right)$ & 4,2 & $-2,8$ & 1,4 & $-0,64$ & 1,26 \\
$\mathrm{RA}\left({ }^{\circ}\right)$ & 10,1 & $-1,8$ & 8,3 & 2,16 & 3,88 \\
TA $\left({ }^{\circ}\right)$ & 2,8 & $-1,3$ & 1,5 & 0,05 & 0,8 \\
$\mathrm{PNA}(\%)$ & 14,44 & 40,41 & 54,85 & 48,61 & 2,92 \\
PA $(\%)$ & 14,44 & 45,14 & 59,58 & 51,39 & 2,92 \\
XNA(mm) & 8,75 & 51,86 & 60,61 & 55,77 & 1,91 \\
YNA(mm) & 65,85 & 66,43 & 132,2 & 95,99 & 19,09 \\
XA(mm) & 7,86 & 26,01 & 33,88 & 28,92 & 1,56 \\
YA(mm) & 46,86 & 113,29 & 160,15 & 138,7 & 14,14 \\
XNA(mm) & 12 & -6 & 6 & 0 & 4 \\
YNA(mm) & 4,7 & -2 & 2,7 & $-0,11$ & 1,49 \\
\hline
\end{tabular}

Se tienen 5600 datos de cada variable. VAR es la variable medida, $\overline{\boldsymbol{X}}$ es el estimado de la media, S la desviación estándar, ANGULO ubicación angular del encaje de la prótesis, $\mathrm{C}$ ángulo de la cadera, $\mathrm{R}$ ángulo de la rodilla, $\mathrm{T}$ ángulo del tobillo, $\mathrm{P}$ porcentaje de soporte de peso corporal, $\mathrm{X}$ ubicación COP eje medial-lateral, Y ubicación COP eje antero-posterior, A ipsilateral, NA contralateral.

\section{E. Metodología para la obtención del modelo de alineación estática}

El modelo de alineación estática de prótesis transtibiales se basó en el principio de teoría de la información (TI) expuesto por Shannon [14], [15], ya que con la TI se puede medir la cantidad de información contenida en los datos (información mutua $\mathrm{I}(\mathrm{X}: \mathrm{Y}))$, el ruido $(\mathrm{H}(\mathrm{Y} \mid \mathrm{X}))$ y la equivocación $(\mathrm{H}(\mathrm{X} \mid \mathrm{Y}))$, lo cual permite hacer un análisis donde se observan las relaciones entre variables, además de mostrar la explicación o predicción del comportamiento de éstas. Adicional, en la TI no se asume ningún tipo de relación en la información contenida en los datos. La Fig. 2 muestra el esquema general propuesto para determinar el modelo.

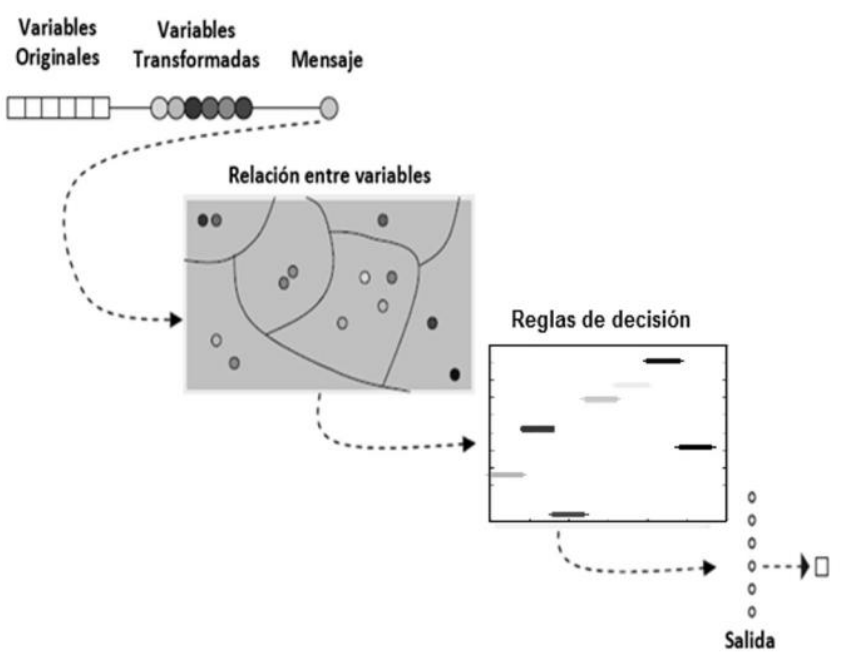

Fig. 2. Esquema general de la metodología propuesta para la obtención del modelo de la alineación estática de prótesis transtibiales.

Las variables originales son los datos recolectados y etiquetados en el estudio descriptivo. Estos datos sufren un proceso de agrupación, ya que para hacer uso de la TI en el análisis de un sistema las variables deben ser categóricas. Al ser las variables de este estudio de tipo numéricas, puesto que provienen de los sensores, se hace un proceso de transformación a categóricas, además por la cantidad de datos que se tienen debe realizarse una reducción del número de éstos. Se hace uso de la técnica Menor Pérdida de Información (LIL, por sus siglas en inglés). Aunque se presenta pérdida de información con esta operación, LIL permite tener en cuenta la variable dependiente para encontrar los puntos de corte, es decir considera el efecto que la partición provoca sobre la variable de salida, de esta forma se tienen categorías que revelan la máxima cantidad de información sobre la salida. La reducción de datos con la determinación del número de categorías obtiene una representación reducida del conjunto de datos menor en volumen pero que produce los mismos resultados analíticos.

Para establecer la relación entre la variable independiente y las dependientes se hace selección de variables, donde se analiza la cantidad de información trasmitida a partir de los datos disponibles, la relación señal/ruido y que las variables seleccionadas no sean colineales. La relación entre las variables en donde a partir del análisis gráfico permite generar las reglas de decisión dando como resultado el modelo de alineación. 
En la construcción y validación del modelo se siguió la metodología de validación cruzada, donde se cuentan con dos conjuntos, uno para la construcción y entrenamiento del modelo y otro de prueba evaluar su rendimiento. Como se dijo, durante la medición se recolectaron tres conjuntos de datos a cada sujeto, se utilizó un conjunto para modelo y entrenamiento y dos conjuntos, mediciones dos y tres, para evaluación de rendimiento.

\section{RESUltados Y DiscusióN}

\section{A. Variación de rangos articulares y $\mathrm{COP}$ con el encaje en alineación}

El análisis de las variables biomecánicas en el estado inicial de la prótesis, es decir, en alineación con ángulo de encaje de $0^{\circ}$, mostró diferencias significantes entre ipsilateral y contralateral para la mayoría de los parámetros medidos. Los valores promedio de los parámetros observados indican que la distribución de peso sobre cada pie es cerca del 50\%, mostrando adecuada distribución de carga sobre cada segmento inferior, igual que lo obtenido en estudios anteriores [16], [17], pero se presenta variabilidad de distribución de peso entre las extremidades.

El COP del lado amputado se encuentra medial y anterior al contralateral, acorde con [16]. Existe movimiento del COP en ambas extremidades en las direcciones medial-lateral y anteroposterior, comprobando que la bipedestación no es un estado estático [18]-[20]. La valoración de los cambios en el COP se realiza con el uso de la desviación estándar. La actividad de la extremidad contralateral en la dirección antero-posterior fue significativamente más alta comparada con el lado amputado, dicha conclusión está coherente con [16] y [21]. El movimiento medial-lateral del COP es bajo, siendo el lado amputado el de mayor variación en este plano. Por el movimiento del COP el sujeto debe compensar para no perder estabilidad, lo cual realiza con la posición de las articulaciones, de allí que la mayor variación en las articulaciones se presente en las rodillas, la otra articulación con alta variabilidad es el tobillo contralateral, lado donde se presenta la mayor variación del COP [19], [20]. La menor variación se da en el tobillo ipsilateral, confirmando su poca contribución a la estabilidad postural. Con respecto a la cadera, la contribución es mayor en el lado amputado.

\section{B. Afectación de la posición angular del encaje sobre los parámetros biomecánicos}

Los cambios de alineación del encaje en el plano sagital influyen en la comodidad del amputado cuando se encuentra en bipedestación, afectando parámetros biomecánicos del amputado. Se confirmó la relación existente entre la ubicación del encaje y las variables biomecánicas incluidas en la investigación. En los rangos articulares se determina que los sujetos ajustan las articulaciones cuando ocurre una alineación incorrecta, siendo el tobillo contralateral el de mayor afectación cuando el encaje está en extensión, la rodilla de este lado también presenta alta afectación, este reporte concuerda con [22] y [9]. Con el encaje en flexión la rodilla ipsilateral es donde mayor alteración se tiene seguido del tobillo contralateral, pero las articulaciones con mayor índice de variación son las caderas. En contralateral, las articulaciones de rodilla y tobillo están en flexión sin tener en cuenta la ubicación del encaje; el comportamiento de la articulación de la cadera varía dependiendo del ángulo del encaje. En ipsilateral, tanto la rodilla como el tobillo siguen los movimientos del encaje, se debe anotar que el movimiento del tobillo es mínimo, menos de un grado; en el caso de la cadera, su posición angular se altera según la posición del ángulo del encaje. Las variaciones de los ángulos de las articulaciones se deben a los mecanismos de control de estabilidad que realizan los pacientes para no perder el equilibrio y caerse, entonces el tobillo contralateral es el que más aporte hace al mecanismo de compensación cuando el encaje está en extensión, y en flexión es la rodilla contralateral.

En la posición del COP, el análisis mostró alta variabilidad de los datos, teniendo mayor afectación en el plano sagital, movimiento antero-posterior. Con el encaje en extensión la ubicación del COP en el eje antero-posterior del lado contralateral presenta el mayor desplazamiento, posicionándose posterior en comparación al punto cuando la prótesis está alineada, caso contrario en el lado ipsilateral que se desplaza anterior con respecto a la posición en alineación. Si el encaje está desalineado en flexión el eje antero-posterior del COP del lado amputado presenta el mayor coeficiente de variación y se desplaza posterior, en el contralateral la ubicación depende del ángulo del encaje. Las mediciones del COP en el eje mediallateral no muestran diferencias significativas en ninguna de las dos extremidades. Estos hallazgos reafirman lo observado por [16].

La distribución de peso entre las extremidades inferiores se ve afectada en forma mínima por la ubicación del encaje, a partir de esto, el encaje en flexión se presenta mayor variabilidad en estos parámetros que cuando se coloca en extensión.

\section{Análisis estadístico de las variables}

La exploración de los datos muestra que todas las variables presentan distribución multimodal y que no existe relación lineal entra la variable de entrada y las variables de salida.

$\mathrm{Al}$ aplicar LIL el número de datos distintos en cada variable se redujo considerablemente, los valores mínimos y máximos de algunas variables se vieron afectados y por tanto el valor medio, Tabla II. El valor de confianza, entendido como la probabilidad de que la distribución de valores de una variable sea representativa de la población, arrojado por cada variable es superior al 96\%, lo cual indica que hay excelente representatividad de la población. 
TABLA II

ESTADÍSTICOS DE LOS DATOS AGRUPADOS

\begin{tabular}{lccccc}
\hline \hline \multicolumn{1}{c}{ VAR } & Dis & Mín & Máx & $\overline{\boldsymbol{X}}$ & $\begin{array}{c}\text { CON } \\
(\%)\end{array}$ \\
\hline ANGULO $\left(^{\circ}\right)$ & 7 & -6 & 6 & 0,014 & 96 \\
CNA $\left({ }^{\circ}\right)$ & 5 & -2 & 2 & 0,061 & 97 \\
RNA $\left(^{\circ}\right)$ & 6 & -1 & 4 & 1,771 & 97 \\
TNA $\left(^{\circ}\right)$ & 6 & 0 & 6 & 2,913 & 97 \\
CDA $\left({ }^{\circ}\right)$ & 3 & -2 & 1 & $-0,43$ & 97 \\
RA $\left({ }^{\circ}\right)$ & 6 & -1 & 8 & 2,33 & 97 \\
TA $\left({ }^{\circ}\right)$ & 3 & -1 & 1 & $-0,02$ & 97 \\
PNA(\%) & 15 & 40 & 54 & 48,11 & 96 \\
PA(\%) & 15 & 45 & 59 & 50,88 & 96 \\
XNA(mm) & 10 & 51 & 60 & 55,29 & 96 \\
YNA(mm) & 62 & 66 & 132 & 95,36 & 96 \\
XA(mm) & 8 & 26 & 33 & 28,46 & 97 \\
YA(mm) & 33 & 113 & 160 & 138,06 & 96 \\
XNA(mm) & 7 & -6 & 6 & 0,014 & 96 \\
YNA(mm) & 5 & -2 & 2 & 0,061 & 97 \\
\hline
\end{tabular}

Estadísticos de los datos luego de la agrupación haciendo uso de la técnica de Menor Pérdida de Información (LIL, por sus siglas en inglés). Se tiene el nombre de las variables (VAR), la cantidad de valores distintos en cada variable (Dis), el valor mínimo (Mín), el máximo (Máx), el estimado de la media $(\boldsymbol{X})$ y el porcentaje de confianza $(\mathrm{CON})$

\section{Selección de variables}

Las variables PNA y PA son complementarias, ya que cada una de ellas soporta un porcentaje del total del peso corporal, luego entre las dos deben sumar $100 \%$, por lo que se utilizó solo una de estas variables (PNA) en la construcción del modelo.

En la Tabla III se observa la relación entre la variable ANGULO y cada una de las salidas, también que los datos transmiten suficiente información sobre las variables a pesar del ruido contenido y se puede obtener un modelo representativo del comportamiento del sistema. Se comprueba que la variación angular de las articulaciones está relacionada con la variación de la ubicación angular del encaje, ya que la información transmitida es superior al $84 \%$ en todos los casos. La relación entre la variación del COP en el eje medial-lateral y ANGULO es baja, cercana a 50\% comprobando que la ubicación del COP en este eje se afecta mínimo con la ubicación del encaje. ANGULO y COP eje antero-posterior se relacionan por encima del $72 \%$, mostrando la fuerte incidencia de la ubicación del encaje sobre el COP en el eje descrito, siendo mayor en el lado amputado. En cuanto a la distribución de peso sobre cada pie, la variable ANGULO presenta poca afectación, 56\%.

\section{E. Obtención y validación del modelo}

El comportamiento de los datos revela la existencia de relaciones disjuntas entre la entrada y las salidas, que se pueden establecer a través de reglas de decisión. Por lo anterior se obtuvo un modelo basado en reglas de decisión, de tipo if..then... con una variable de entrada (ANGULO) y once salidas.
TABLA III

DATOS INFOMÉTRICOS DE LAS VARIABLES

\begin{tabular}{lccc}
\hline \hline $\begin{array}{l}\text { VARIABLE } \\
\text { SALIDA }\end{array}$ & $\mathrm{I}(\mathrm{X}: \mathrm{Y})$ & $\mathrm{H}(\mathrm{Y} \mid \mathrm{X})$ & $\mathrm{H}(\mathrm{X} \mid \mathrm{Y})$ \\
\hline $\mathrm{CNA}\left({ }^{\circ}\right)$ & 85 & 0,3 & 1,14 \\
$\mathrm{RNA}^{\circ}\left(^{\circ}\right.$ & 84 & 0,34 & 1,06 \\
$\left.\mathrm{TNA}^{\circ}\right)$ & 91 & 0,19 & 0,77 \\
$\mathrm{CDA}\left({ }^{\circ}\right)$ & 100 & 0 & 0,75 \\
$\mathrm{RA}\left({ }^{\circ}\right)$ & 95 & 0,1 & 0,39 \\
$\mathrm{TA}\left({ }^{\circ}\right)$ & 92 & 0,1 & 1,42 \\
$\mathrm{PNA}(\%)$ & 55,73 & 1,44 & 0,99 \\
$\mathrm{XNA}(\mathrm{mm})$ & 51,2 & 1,34 & 1,4 \\
$\mathrm{YNA}(\mathrm{mm})$ & 72 & 0,87 & 0,57 \\
$\mathrm{XA}(\mathrm{mm})$ & 61,4 & 0,9 & 1,38 \\
$\mathrm{YA}(\mathrm{mm})$ & 84 & 0,51 & 0,12 \\
XNA(mm) & 85 & 0,3 & 1,14 \\
YNA(mm) & 84 & 0,34 & 1,06 \\
\hline
\end{tabular}

Resumen de los datos infométricos de las variables. Los valores mostrados son porcentajes de relación (información mutua $\mathrm{I}(\mathrm{X}: \mathrm{Y})$ ), el ruido $(\mathrm{H}(\mathrm{Y} \mid \mathrm{X})$ ) y la equivocación $(\mathrm{H}(\mathrm{X} \mid \mathrm{Y}))$, entre la variable de entrada ANGULO y cada salida.

El rendimiento del modelo se evidenció mediante la comparación de los valores pronosticados por el modelo de alineación y los datos experimentales recogidos en las mediciones 2 y 3 . La comparación entre los resultados experimentales y simulados se muestra en la Fig. 3, la línea continua es la predicción del modelo y las otras líneas son los datos experimentales de las tres mediciones. Se observa que los valores simulados y experimentales coinciden.

A pesar de las conclusiones obtenidas por inspección visual del comportamiento del modelo, este fue evaluado con pruebas no paramétricas para observar su desempeño. Se calculó la bondad de ajuste de Kolmogorov-Smirnov y la divergencia de Kullback-Leibler. La Tabla IV muestra que el rendimiento del modelo es superior al 94\% según la prueba de KolmogorovSmirnov (KS) aplicada a los datos de entrenamiento, en los datos de prueba el rendimiento es superior al $93 \%$ y en la validación al $84 \%$. Al observar el rendimiento con la divergencia de Kullback-Leibler (KL), se tiene que para el grupo de datos de validación de la variable Tobillo Amputado la longitud de la distancia es 0.36 bits, para todos los demás casos es 0 bits. 

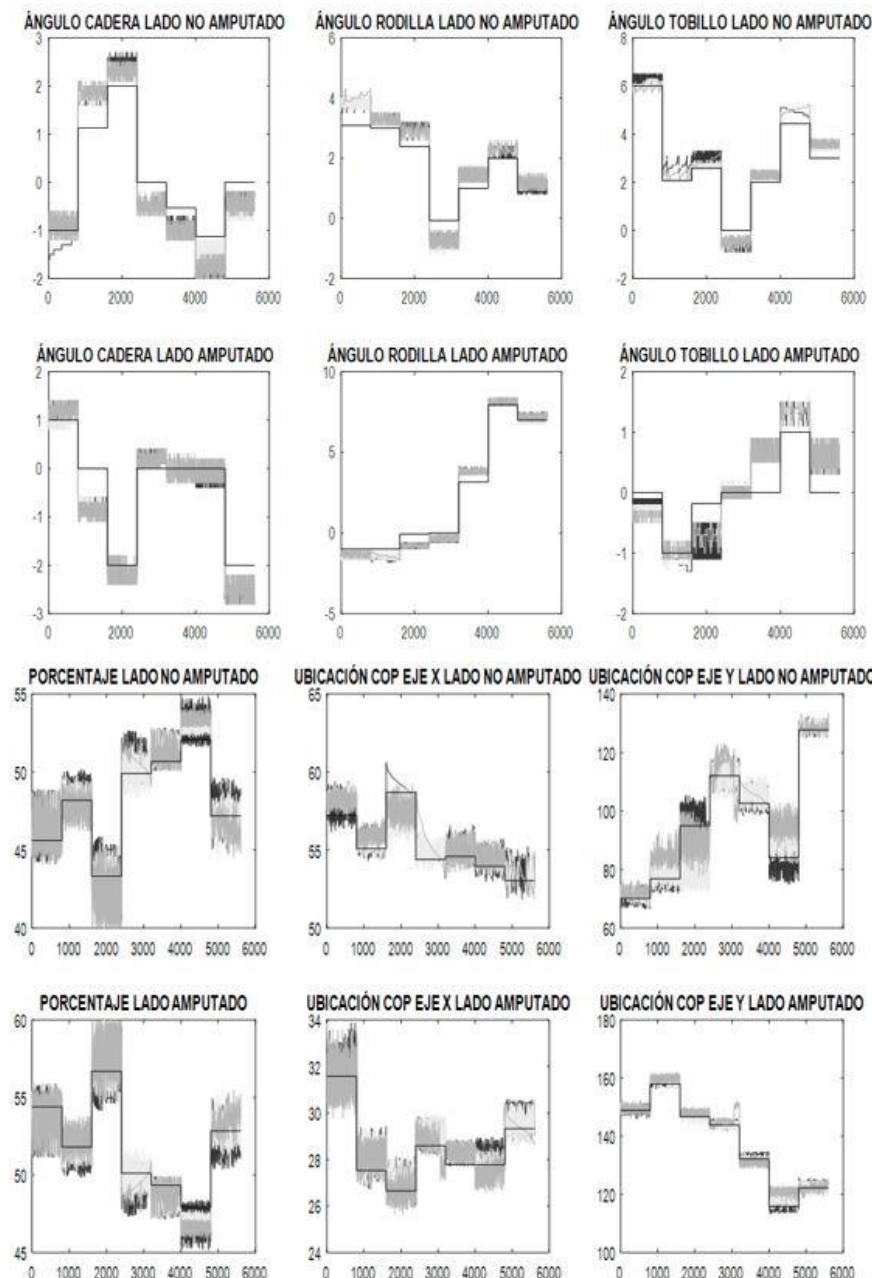

Fig. 3. Gráficas de la predicción del modelo en comparación con las mediciones. La línea continua es la predicción del modelo, las demás líneas corresponden a los datos obtenidos experimentalmente en los tres momentos de medición.

Con los resultados de la prueba KS se observa que el modelo concuerda con los datos experimentales, luego este modelo es válido para representar el sistema estudiado. El valor de las distancias obtenido al calcular la divergencia KL muestra la similitud entre las funciones de distribución del modelo estocástico obtenido y los datos experimentales, es decir los datos se ajustan correctamente al modelo.

\section{CONCLUSIONES}

Se estableció una relación entre variables biomecánicas (ángulos articulares, ubicación del COP y distribución plantar) y la alineación protésica. La selección de las variables mencionadas se debe a que la bipedestación es una posición inestable que requiere regulación constante que involucra movimiento de las articulaciones, variación del COP y distribución de peso, entre otros. Se incluyeron las dos extremidades inferiores porque se ha comprobado la existencia de interacción de la extremidad contralateral en la bipedestación y por tanto en la alineación estática, así el monitoreo de ambas piernas simultáneamente es esencial para determinar el efecto combinado de la alineación sobre los parámetros biomecánicos presentes en bipedestación.
TABLA IV

ERROR CALCULADO

\begin{tabular}{|c|c|c|c|}
\hline VARIABLE & DATO & KL(bits) & $\mathrm{KS}(\%)$ \\
\hline \multirow[t]{4}{*}{ CNA } & $\mathrm{E}$ & 0 & 100 \\
\hline & PRUEBA & 0 & 100 \\
\hline & M2 & 0 & 100 \\
\hline & M3 & 0,001 & 100 \\
\hline \multirow[t]{4}{*}{ RNA } & $\mathrm{E}$ & 0 & 100 \\
\hline & PRUEBA & 0 & 100 \\
\hline & M2 & 0 & 100 \\
\hline & M3 & 0,005 & 100 \\
\hline \multirow[t]{4}{*}{ TNA } & E & 0 & 98,8 \\
\hline & PRUEBA & 0 & 97,55 \\
\hline & M2 & 0 & 99,5 \\
\hline & M3 & 0,001 & 98,248 \\
\hline \multirow[t]{4}{*}{ CDA } & $\mathrm{E}$ & 0 & 100 \\
\hline & PRUEBA & 0 & 100 \\
\hline & M2 & 0 & 98,934 \\
\hline & M3 & 0 & 95,833 \\
\hline \multirow[t]{4}{*}{ RA } & E & 0 & 100 \\
\hline & PRUEBA & 0 & 99,97 \\
\hline & M2 & 0 & 99,97 \\
\hline & M3 & 0 & 99,97 \\
\hline \multirow[t]{4}{*}{ TA } & $\mathrm{E}$ & 0 & 98,59 \\
\hline & PRUEBA & 0 & 97,7 \\
\hline & M2 & 0,36 & 100 \\
\hline & M3 & 0,002 & 100 \\
\hline \multirow[t]{4}{*}{ PNA } & $\mathrm{E}$ & 0 & 97,4 \\
\hline & PRUEBA & 0 & 97,3 \\
\hline & M2 & 0 & 97,5 \\
\hline & M3 & 0 & 98,7 \\
\hline \multirow[t]{4}{*}{ XA } & E & 0 & 94,47 \\
\hline & PRUEBA & 0 & 93,85 \\
\hline & M2 & 0 & 94,93 \\
\hline & M3 & 0,002 & 94,52 \\
\hline \multirow[t]{4}{*}{ YA } & $\mathrm{E}$ & 0 & 100 \\
\hline & PRUEBA & 0 & 99,98 \\
\hline & M2 & 0 & 99,99 \\
\hline & M3 & 0,001 & 99,98 \\
\hline \multirow[t]{4}{*}{ XNA } & E & 0 & 96,5 \\
\hline & PRUEBA & 0 & 96,3 \\
\hline & M2 & 0 & 95,7 \\
\hline & M3 & 0,001 & 88,8 \\
\hline \multirow[t]{4}{*}{ YNA } & E & 0 & 96,5 \\
\hline & PRUEBA & 0 & 96,7 \\
\hline & M2 & 0 & 99 \\
\hline & M3 & 0,001 & 99,6 \\
\hline
\end{tabular}

Se utilizaron las pruebas estadísticas de Kullback-Leibler (KL) y Kolmogorov-Smirnov (KS) para observar el ajuste entre las distribuciones de los datos de la predicción arrojada por el modelo y cada una de las mediciones realizadas. Se calculó el error al grupo de entrenamiento (E), prueba (medición 1), medición 2 (M2) y medición 3 (M3).

Se consolidó una base de datos de la alineación estática que incluye variables cinemáticas y cinéticas de ángulos de articulaciones, distribución de peso corporal en las extremidades inferiores y ubicación del COP.

Es evidente la asimetría del comportamiento entre la extremidad no amputada y la amputada. Se observa variabilidad del COP bajo ambos pies, la cual es mayor en contralateral con respecto a ipsilateral, siendo el movimiento en el eje anteroposterior mayor que en el eje medial-lateral. Es razonable que la extremidad no amputada presente menor estabilidad que la amputada con prótesis, debido a que tiene más musculatura, propiocepción y control motor lo que incide en un mayor rango de movimiento en cada uno de sus segmentos (es decir, el pie intacto y el tobillo se pueden mover y ajustar para ayudar a la 
persona a mantener su estabilidad). La distribución del peso corporal sobre cada una de las extremidades se encuentra cerca del $50 \%$ del peso, mostrando que las personas amputadas tienden a equilibrar la distribución de peso para mantener estabilidad.

Las alteraciones del COP y rangos articulares al variar el ángulo del encaje muestran que al afectar la alineación de la prótesis en el plano sagital, el amputado transtibial compensa mediante el movimiento de las articulaciones sobre el centro de presión de los pies obteniendo equilibrio.

El uso de la teoría de la información en la obtención del modelo predictivo hace que se tenga la mayor cantidad de información de la entrada, también permite reducir el ruido y muestra la proyección de aproximación del modelo sin necesidad de construirlo. Así, se pudo conocer de antemano que los datos eran suficientes para predecir las variables de salida.

El modelo de predicción propuesto permite pronosticar la consecuencia de una inadecuada alineación del encaje de una prótesis transtibial sobre parámetros biomecánicos. El modelo es una serie de reglas de decisión fundamentadas en la información que transmite, lo que permite manejar datos categóricos y multimodales y es robusto. La funcionalidad del modelo se validó con las pruebas estadísticas de KolmogorovSmirnov (KS) y Kullback-Leibler (KL), contrastando la predicción del modelo y los datos de las mediciones en el segundo y tercer registro. Las pruebas arrojaron que los datos de las mediciones se ajustan a las predicciones del modelo (KS superior a $84 \%$ y KL inferior a 0.36 bits). Se concluye que el modelo muestra la afectación de la ubicación del encaje de la prótesis sobre variables biomecánicas de un amputado transtibial usuario de prótesis.

\section{REFERENCIAS}

[1] M. Leblanc, Give Hope - Give a Hand - The LN-4 Prosthetic Hand [en línea]. California: Stanford University, 2008. Disponible en: http://www.stanford.edu/class/engr110/2011/LeBlanc-03a.pdf.

[2] E. Strait, Prosthetics in Developing Countries [en línea]. Prosthetic Resident, $2006 . \quad$ Disponible en: http://www.oandp.org/publications/resident/pdf/DevelopingCountries.pd f.

[3] Dirección Contra Minas, Víctimas de Minas Antipersonal [en línea]. Colombia: Dirección contra minas. Disponible en: http://www.accioncontraminas.gov.co/estadisticas/Paginas/victimasminas-antipersonal.aspx.

[4] E. Camargo, L. A. Luengas, and M. Balaguera, "Respuesta a carga de una prótesis transtibial con elementos infinitos durante el apoyo y balanceo," Visión Electrónica, vol. 6, pp. 82-92, 2012.

[5] M. S. Zahedi, W. D. Spence, S. E. Solomonidis, and J. P. Paul, "Alignment of lower-limb prostheses.," J. Rehabil. Res. Dev., vol. 23, no. 2, pp. 2-19, Apr. 1986.

[6] L. Yang, S. E. Solomonidis, W. D. Spence, and J. P. Paul, "The influence of limb alignment on the gait of above-knee amputees," J. Biomech., vol. 24, no. 2, pp. 981-997, 1991. DOI: 10.1016/0021-9290(91)90016-G

[7] T. Kobayashi, M. S. Orendurff, M. Zhang, and D. Boone, "Effect of alignment changes on sagittal and coronal socket reaction moment interactions in transtibial prostheses.," J. Biomech., vol. 46, no. 7, pp. 1343-50, Apr. 2013. DOI: 10.1016/j.jbiomech.2013.01.026

[8] S. Blumentritt, T. Schmalz, and R. Jarasch, "Significance of static prosthesis alignment for standing and walking of patients with lower limb amputation," Orthopade, vol. 30, no. 3, pp. 161-168, 2001. DOI: $10.1007 / \mathrm{s} 001320050590$

[9] J. Xiaohong, L. Xiaobing, D. Peng, and Z. Ming, "The Influence of Dynamic Trans-tibial Prosthetic Alignment on Standing Plantar Foot
Pressure.," Conf. Proc. IEEE Eng. Med. Biol. Soc., vol. 7, pp. 6916-8, Jan. 2005. DOI: 10.1109/IEMBS.2005.1616096

[10] J. R. Engsberg, T. L. Allinger, and J. A. Harder, "Standing pressure distribution for normal and below-knee amputee children.," Prosthet. Orthot. Int., vol. 13, no. 3, pp. 152-155, 1989. DOI: $10.3109 / 03093648909079425$.

[11] Biometrics Ltd, Biometrics Ltd. [en línea]. Reino Unido: Biometrics, 2015. Disponible en: http://www.biometricsltd.com/.

[12] Novel.de, The pedar system [en línea]. Alemania: Novel. Disponible en: http://www.novel.de/novelcontent/pedar

[13] M. Lord and D. M. Smith, "Foot loading in amputee stance.," Prosthet. Orthot. Int., vol. 8, no. 3, pp. 159-64, Dec. 1984. DOI: $10.3109 / 03093648409146079$

[14] C. E. Shannon, "A mathematical theory of communication," Bell Syst. Tech. J., vol. 27, pp. 379-423, July 1948, DOI: 10.1002/j.15387305.1948.tb00917.x

[15] E. Z. Varela, S. V. Barake, C. Flórez, and F. De la Cruz, "Cibernética: Fundamentos y evolución," Ingeniería y Desarrollo, vol. 7, pp. 14-20, 2000.

[16] E. Isakov, J. Mizrahi, Z. Susak, I. Ona, and N. Hakim, "Influence of prosthesis alignment on the standing balance of below-knee amputees," Clin. Biomech., vol. 9, no. 4, pp. 258-262, 1994. DOI: 10.1016/02680033(94)90008-6

[17] J. Viton, L. Mouchnino, M. L. Mille, M. Cincera, A. Delarque, A. Pedotti, A. Bardot, and J. Massion, "Equilibrium and movement control strategies in trans-tibial amputees," Prosthet. Orthot. Int., vol. 24, no. 2, pp. 108116, Jan. 2000. DOI: 10.1080/03093640008726533

[18] S. Onyshko and D. A. Winter, "A mathematical model for the dynamics of human locomotion," J. Biomech., vol. 13, no. 4, pp. 361-368, 1980, DOI: 10.1016/0021-9290(80)90016-0

[19] W. H. Gage, D. A. Winter, J. S. Frank, and A. L. Adkin, "Kinematic and kinetic validity of the inverted pendulum model in quiet standing.," Gait Posture, vol. 19, no. 2, pp. 124-32, 2004. DOI: 10.1016/S09666362(03)00037-7

[20] D. Winter, F. Prince, J. S. Frank, C. Powell, and K. F. Zabjek, "Unified theory regarding $\mathrm{A} / \mathrm{P}$ and $\mathrm{M} / \mathrm{L}$ balance in quiet stance," J. Neurophysiol., vol. 75, no. 6, pp. 2334-2343, 1996.

[21] C. Kendell, E. D. Lemaire, N. L. Dudek, and J. Kofman, "Indicators of dynamic stability in transtibial prosthesis users.," Gait Posture, vol. 31, no. 3, pp. 375-379, 2010. DOI: 10.1016/j.gaitpost.2010.01.003.

[22] E. Isakov, J. Mizrahi, H. Ring, Z. Susak, and N. Hakim, "Standing sway and weight-bearing distribution in people with below-knee amputations.,", Arch. Phys. Med. Rehabil., vol. 73, no. 2, pp. 174-178, 1992. DOI: 10.5555/uri:pii:000399939290097G.

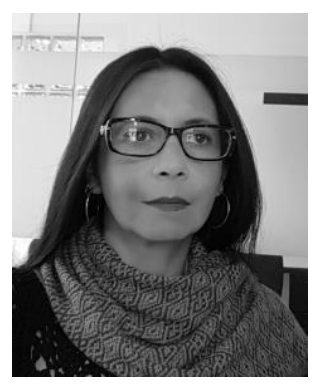

Lely Adriana Luengas Contreras, nació en Bogotá, Colombia en 1970. Se graduó como Ingeniera Electrónica de la Universidad Autónoma de Colombia, Bogotá, Colombia, en 1997; obtuvo el grado de Magíster en Ingeniería Eléctrica de la Universidad de Los Andes, Bogotá, Colombia, en 1999; y el grado de Doctora en Ingeniería (PhD) de la Pontificia Universidad Javeriana, Bogotá, Colombia, en 2016. Se desempeñó como Coordinadora de Investigación en la Universidad de San Buenaventura, Bogotá, Colombia. En 2007 se vinculó a la Universidad Distrital Francisco José de Caldas, donde trabaja actualmente como docente e investigadora. Su área de investigación es Bioingeniería, específicamente análisis de movimiento humano. Ha publicado cerca de 20 artículos científicos en diferentes revistas científicas y es autora de cuatro libros.

Fue Condecorada con la orden al Mérito por el Senado de la República de Colombia por sus aportes a la Nación en el área de Bioingeniería.

ORCID: http://orcid.org/0000-0002-3600-4666 


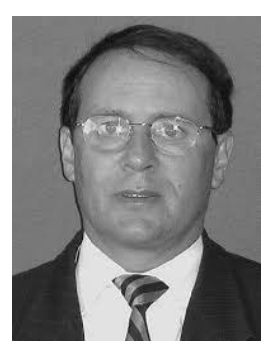

Pedro Raúl Vizcaya Guarín, nació en Bogotá, Colombia. Obtuvo el título de Ingeniero Electrónico en 1980 en la Pontificia Universidad Javeriana, Bogotá, Colombia. En 1997 obtuvo Master Engenharia de Eletricidade, Instituto Politécnico Rensselaer, Estados Unidos de América. En 1998 obtiene PhD Electrical Engineering en Instituto Politécnico Rensselaer, Estados Unidos de América. Es docente e investigador en la Pontificia Universidad Javeriana, Bogotá, Colombia.

ORCID: https://orcid.org/0000-0002-9164-5124

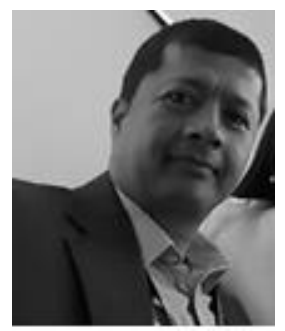

Giovanni Sánchez Prieto, nació en Bogotá, Colombia en 1973. Se graduó como Ingeniero Electrónico de la Universidad Autónoma de Colombia, Bogotá, Colombia, en 1997; obtuvo el grado de Especialista en pedagogía y docencia Universitaria en Universidad de San Buenaventura, Bogotá, Colombia, en 2002. Es Director de Tecnologías en la Universidad de San Buenaventura, Bogotá, Colombia. Ha publicado varios artículos científicos en Bioingeniería.

ORCID: http://orcid.org/0000-0003-2108-6491 